\title{
Real-Time Quaking-Induced Conversion Detection of Bovine Spongiform Encephalopathy Prions in a Subclinical Steer
}

\author{
Soyoun Hwang', M. Heather West Greenlee'2, Anne Balkema-Buschmann ${ }^{3}$, \\ Martin H. Groschup ${ }^{3}$, Eric M. Nicholson ${ }^{1}$ and Justin J. Greenlee ${ }^{1 *}$ \\ ${ }^{1}$ United States Department of Agriculture, Agricultural Research Service, National Animal Disease Center, Virus and Prion \\ Research Unit, Ames, IA, United States, ${ }^{2}$ Department of Biomedical Sciences, lowa State University College of Veterinary \\ Medicine, Ames, IA, United States, ${ }^{3}$ Institute of Novel and Emerging Infectious Diseases, Friedrich-Loeffler-Institut, Federal \\ Research Institute for Animal Health, Greifswald, Germany
}

\section{OPEN ACCESS}

Edited by:

Francisco Javier Salguero, University of Surrey, United Kingdom

Reviewed by: Enric Vidal,

IRTA-Centre for Research on Animal Health (CReSA), Spain Juan Carlos Espinosa Martín, Instituto Nacional de Investigación y Tecnología Agraria y Alimentaria (INIA), Spain

*Correspondence: Justin J. Greenlee justin.greenlee@ars.usda.gov

Specialty section: This article was submitted to Veterinary Experimental and

Diagnostic Pathology,

a section of the journal Frontiers in Veterinary Science

Received: 29 September 2017 Accepted: 20 December 2017

Published: 19 January 2018

Citation: Hwang S, West Greenlee MH, Balkema-Buschmann A, Groschup MH, Nicholson EM and Greenlee JJ (2018) Real-Time Quaking-Induced Conversion

Detection of Bovine Spongiform Encephalopathy Prions in a Subclinical Steer.

Front. Vet. Sci. 4:242. doi: 10.3389/fvets.2017.00242
Bovine spongiform encephalopathy (BSE) belongs to a group of fatal prion diseases that result from the misfolding of the cellular prion protein $\left(\mathrm{PrP}^{\mathrm{C}}\right)$ into a pathogenic form ( $\left.\mathrm{PrPs}^{\mathrm{Sc}}\right)$ that accumulates in the brain. In vitro assays such as serial protein misfolding amplification and real-time quaking-induced conversion (RT-QulC) allow assessment of the conversion of $\mathrm{PrP}^{\mathrm{c}}$ to $\mathrm{PrP}^{\mathrm{sc}}$. RT-QulC can be used for the detection of prions in a variety of biological tissues from humans and animals. However, there is no such comparison of RT-QuIC data between BSE positive and presymptomatic cattle. Further, the current study assesses prion distribution in multiple brain regions of clinically ill or subclinical animals. Here, we compare RT-QulC reactions seeded with brain samples collected from experimentally inoculated cattle that were clinically ill or subclinically affected with BSE. The results demonstrate RT-QulC seeding in various brain regions of an animal with subclinical BSE despite being determined negative by immunohistochemistry. Bioassay of the subclinical animal and RT-QuIC of brainstem from inoculated knockout (PRNP ${ }^{-I^{-}}$) cattle were used to confirm infectivity in the subclinical animal and determine that RT-QulC reactions were not the result of residual inoculum, respectively. These results confirm that RT-QulC is a highly sensitive prion detection assay that can detect prions in a steer prior to the onset of clinical signs of BSE.

\footnotetext{
Keywords: bovine spongiform encephalopathy, diagnosis, prion diseases, prion protein, protein misfolding, realtime quaking-induced conversion, transmissible spongiform encephalopathy
}

\section{INTRODUCTION}

Bovine spongiform encephalopathy (BSE) belongs to a group of fatal neurologic diseases that result from the misfolding of the cellular prion protein $\left(\mathrm{PrP}^{\mathrm{C}}\right)$ into a pathogenic form $\left(\operatorname{PrP}^{\mathrm{S} c}\right)$ in the brain (1-3). Collectively, these diseases are referred to as prion diseases or transmissible spongiform encephalopathies (TSEs). In addition to BSE, the prion diseases include scrapie in sheep, chronic wasting disease (CWD) in cervids, and Creutzfeldt-Jakob disease (CJD), fatal familial insomnia, Gerstmann-Sträussler-Scheinker syndrome, and kuru in humans. Classical BSE is a feedborne disease in cattle and transmits to humans as variant CJD $(4,5)$. 
Currently approved BSE diagnostic tests are based on the direct detection of $\mathrm{PrP}^{\mathrm{Sc}}$ by immunoblot, enzyme immunoassay (EIA or ELISA), or immunohistochemistry (IHC) $(6,7)$. New prion detection tools relying on the in vitro amplification of $\mathrm{PrP}^{\mathrm{Sc}}$ have been developed and include protein misfolding cyclic amplification (PMCA) (8-10) and the real-time quakinginduced conversion (RT-QuIC) assay (11-18). Both of these approaches can amplify very low levels of $\mathrm{PrP}^{\mathrm{Sc}}$ to levels that are readily detectable. PMCA is a very useful methodology for sensitive detection of prions in various samples of different species, but it requires an animal derived substrate (usually brain homogenate) and is time consuming because final analysis requires Western blotting. Unlike PMCA, RT-QuIC is a relatively simple technique that makes use of recombinant prion protein as a substrate. This technique monitors the fibril formation in real-time via binding of the fluorescent marker, thioflavin $\mathrm{T}$ (ThT), to the resulting amyloid fibrils. ThT fluorescence increases as the amount of amyloid fibril accumulates through conversion of monomeric $\operatorname{PrP}^{\mathrm{C}}$ to an amyloid conformation. The RT-QuIC assay has been shown to detect low levels of TSE infectivity and to detect human and animal prions in various tissues $(11-18)$.

Even after experimental inoculation by the intracranial route, there is a period of time during incubation where $\operatorname{PrP}^{S c}$ is undetectable in brain samples when assayed by traditional diagnostic methods for prion diseases (19). Amplification based assays, such as RT-QuIC represent an opportunity for evaluation of $\mathrm{PrP}^{\mathrm{Sc}}$ distribution in situations where the level is anticipated to be low, such as during the preclinical incubation period. Currently, there is a lack of RT-QuIC data on detection of prion diseases in subclinical cattle. Here, we applied RT-QuIC to assess relative levels and distribution of $\mathrm{PrP}^{\mathrm{Sc}}$ among cattle in various groups: clinically affected with BSE; BSE inoculated, but without clinical signs of disease (subclinical); inoculated knockouts $\left(P R N P^{-/-}\right)$ that did not develop clinical disease after long incubation periods, and sham-inoculated controls.

\section{MATERIALS AND METHODS}

\section{Ethics Statement}

The laboratory and animal experiments were conducted in Biosafety Level 2 spaces that were inspected and approved for importing prion agents by the US Department of Agriculture, Animal and Plant Health Inspection Service, Veterinary Services. The studies were done in accordance with the Guide for the Care and Use of Laboratory Animals (Institute of Laboratory Animal Resources, National Academy of Sciences, Washington, DC, USA) and the Guide for the Care and Use of Agricultural Animals in Research and Teaching (Federation of Animal Science Societies, Champaign, IL, USA). The protocols were approved by the Institutional Animal Care and Use Committee at the National Animal Disease Center (protocol numbers: 3636 and 3985), which included a biosafety review. Cattle were monitored twice daily for the appearance of clinical signs suggestive of prion disease such as aggression, behavior changes, decreased feed intake, loss of body condition, ataxia, prolonged recumbency, or inability to rise. All inoculated animals developed the clinical signs specifically described in Table 1 and no animals died prior to the experimental endpoint. To ensure a humane endpoint, animals were euthanized if they demonstrated clinical signs of prolonged anorexia $(>24 \mathrm{~h})$ or recumbency $(>12 \mathrm{~h})$. Animals exhibiting behavioral or postural changes consistent with TSE were examined by a veterinarian. Upon veterinary confirmation of unequivocal clinical signs of TSE, affected cattle were killed with an intravenous injection of pentobarbital sodium according to the manufacturer's directions and necropsied. Any other concurrent diseases were treated under the direction of a veterinarian.

\section{Brain Samples from Experimentally Inoculated Cattle}

Cattle $(n=12)$ were intracranially inoculated with $1 \mathrm{ml}$ of a $10 \%$ (wt./vol.) brain homogenate from a cow diagnosed with classical BSE in the US in 2003 as previously described (20). The PRNP gene of all inoculated animals was sequenced. The predicted amino acid sequences from inoculated cattle were similar to each other and consistent with previously reported cattle sequences (21). Brain samples from various regions including brainstem at the level of the obex, cerebellum, midbrain, thalamus, and cerebrum were obtained from cattle in the following experimental groups: (1) wild-type cattle intracranially sham-inoculated with normal brain homogenate (negative controls), (2) wild-type cattle intracranially inoculated with classical BSE (22), or (3) knockout cattle $\left(P R N P^{-/-}\right)$intracranially inoculated with the transmissible mink encephalopathy (TME) agent. Homogenates were prepared from brainstem sections of TSE-free cattle or cattle with clinical signs of TME (23-25) or classical BSE by homogenization with $1.0 \mathrm{~mm}$ silica beads in $1 \times$ Dulbecco's PBS $\mathrm{pH} 7.4$ to produce a $10 \% \mathrm{w} / \mathrm{v}$ homogenate. Samples were centrifuged briefly at $1,000 \mathrm{~g}$ for $2 \mathrm{~min}$ and supernatants were separated and stored at $-80^{\circ} \mathrm{C}$ in aliquots. The sham-inoculated negative control animal was housed separately from the other cattle in this study. All animal donor information including TSE isolate inoculated, source of inoculum, incubation periods, and description of clinical signs are shown in Table 1.

\section{Microscopic Examination and IHC}

After fixation in $10 \%$ neutral buffered formalin, tissues were embedded in paraffin and processed by routine methods for microscopic examination after staining by hematoxylin and eosin or by immunohistochemical methods as described previously (26). Sections of cerebrum, thalamus, midbrain, and brainstem at the level of the obex were assessed for spongiform change and $\mathrm{PrP}^{\mathrm{Sc}}$ accumulation. After deparaffinization and rehydration, sections ( $4 \mu \mathrm{m}$ on charged slides) were autoclaved for $20 \mathrm{~min}$ in antigen retrieval solution (DAKO Target Retrieval Solution, DAKO Crop., Carpinteria, CA, USA), and stained with an indirect, alkaline phosphatase-labeled secondary antibody (ultraview Universal Alkaline Phosphatase Red Detection Kit, Ventana Medical Systems, Inc., Tucson, AZ, USA). The primary antibody was mAb F99/97.6.1 (27) used at $10 \mu \mathrm{g} / \mathrm{ml}$ for a $32 \mathrm{~min}$ incubation at $37^{\circ} \mathrm{C}$. Slides were counterstained with Gill's hematoxylin 
TABLE 1 | Inoculum and clinical history for experimental cattle.

\begin{tabular}{|c|c|c|c|c|c|c|}
\hline Animal number & Inoculum & Source of inoculum & Days postinoculation & Age at death (month) & Signs & Clinical description \\
\hline 1 & Classical BSE & US 2003 (WA) & 939 & 86 & - & No clinical signs \\
\hline 2 & Classical BSE & US 2003 (WA) & 687 & 77 & + & $\begin{array}{l}\text { Sawhorse stance, increased startle } \\
\text { response, goose-stepping gate }\end{array}$ \\
\hline 3 & Classical BSE & US 2003 (WA) & 639 & 76 & + & $\begin{array}{l}\text { Sawhorse stance, irritable when } \\
\text { encouraged to walk, fell down while walking }\end{array}$ \\
\hline 4 & TME & Second passage & 689 & 31.8 & - & No clinical signs \\
\hline 5 & TME & Second passage & 1,433 & 54.3 & - & No clinical signs \\
\hline 6 & Sham & $\mathrm{NBH}$ & 1,667 & 80.7 & - & No clinical signs \\
\hline
\end{tabular}

$\mathrm{NBH}$, normal brain homogenate.

and bluing agent (Ventana Medical Systems, Inc., Tucson, AZ, USA) prior to coverslipping.

\section{Western Blotting of Cattle Brain Homogenates}

All brain tissues including one sham-inoculated negative control, two TME-infected PRNP knock out cattle, three BSE-infected cattle: two with clinical signs (animal No. 2 and No. 3) and one without clinical signs (animal No. 1) were run on the Western blot (WB). Each sample was digested with $50 \mu \mathrm{g}$ of proteinase K (PK) for $1 \mathrm{~h}$ at $42^{\circ} \mathrm{C}$ and separated by SDS-PAGE on $12 \%$ polyacrylamide minigels (Invitrogen) and then transferred onto polyvinylidene difluoride membranes (Millipore, Billerica, MA, USA) for $45 \mathrm{~min}$ at $30 \mathrm{~V}$. Bovine serum albumin (3\%) in Tris-buffered saline was used to block the membranes for $1 \mathrm{~h}$ and then membrane was incubated with mouse anti-PrP monoclonal antibody $6 \mathrm{H} 4$ as the primary antibody at $1: 10,000$ dilution $(0.1 \mu \mathrm{g} / \mathrm{ml})$ for another hour. Then, a biotinylated sheep antimouse secondary antibody at $0.05 \mu \mathrm{g} / \mathrm{ml}$ and a streptavidin-horseradish peroxidase conjugate were used in conjunction with a chemiluminescent detection system (Pierce ECL plus, Thermo Fisher) and visualized on an imaging system capable of detecting luminescence.

\section{EIA of Brain Homogenates from Cattle}

Enzyme immunoassay was performed to determine the relative amount of misfolded protein in brain homogenates from all animals including one sham-inoculated, two TME-infected PRNP knock out cattle, three BSE-infected cattle: two with clinical signs (animal No. 2 and No. 3) and one without clinical signs (animal No. 1) using an IDEXX HerdChek BSE EIA kit without treatment of PK. The cutoff value was determined by the negative control sample provided by the manufacturer and the optical density value was around $0.08 \pm 0.005$. Samples were considered positive if the optical density value was over 0.15 .

\section{PK Sensitivity of Brain Homogenates from Cattle}

Brain homogenates $(10 \% \mathrm{w} / \mathrm{v})$ prepared in PBS were digested or treated with different concentrations of PK $(0-0.5 \mathrm{mg} / \mathrm{ml}$; VWR, Visalia, CA, USA) for $1 \mathrm{~h}$ at $37^{\circ} \mathrm{C} .1 \times$ pefabloc proteinase inhibitor (VWR, Visalia, CA, USA) was added to terminate the reaction. The relative amount of misfolded protein was measured by IDEXX HerdChek BSE Kit (see above). Each sample was performed in triplicate.

\section{Recombinant Prion Protein Production and Purification}

Escherichia coli $[\mathrm{BL} 21(\lambda \mathrm{DE} 3)]$ transformed with the pET28a vector harboring the wild-type bovine PrP gene (amino acids 25-241; GenBank accession number: DQ875147.1) was grown, and the bovine prion protein was expressed and purified as described by Vrentas et al. (28). The concentration of purified pooled protein was measured by UV spectroscopy and calculated from the absorbance at $280 \mathrm{~nm}$ using an extinction coefficient of $63,495 \mathrm{M} / \mathrm{cm}$ as calculated for wild-type protein.

\section{RT-QuIC Protocol}

Real-time quaking-induced conversion reactions were performed as previously described (29). The RT-QuIC reaction buffer was composed of $10 \mathrm{mM}$ phosphate buffer ( $\mathrm{pH} 7.4$ ), $300 \mathrm{mM} \mathrm{NaCl}, 0.1 \mathrm{mg} / \mathrm{ml}$ recombinant bovine prion protein, $10 \mu \mathrm{M}$ ThT, $1 \mathrm{mM}$ ethylenediaminetetraacetic acid tetrasodium salt, and $0.001 \%$ SDS. A volume of $98 \mu \mathrm{l}$ of the RT-QuIC reaction buffer was loaded into each well of a black 96-well plate with a clear bottom (Nunc, Thermo Fisher Scientific) and $2 \mu \mathrm{L}$ of brain homogenate dilution was added to test for seeding activity. Brain homogenate $(10 \% \mathrm{w} / \mathrm{v})$ was prepared as previously described (11) and stored at $-80^{\circ} \mathrm{C}$.

The plate was sealed and incubated in a BMG FLUOstar Omega plate reader at $42^{\circ} \mathrm{C}$ for $100 \mathrm{~h}$ with cycles of $15 \mathrm{~min}$ shaking (700 rpm double orbital pattern) and $15 \mathrm{~min}$ rest. ThT fluorescence measurements (460 nm excitation and $480 \mathrm{~nm}$ emission) were taken every $15 \mathrm{~min}$, with the gain set at 1,400. All reactions for each sample were performed in eight replicates (quadruplicates in two independent RT-QuIC assays). ThT fluorescence data are displayed as the average ThT fluorescence of four technical replicates for each time point and, to be considered positive, the ThT fluorescence of at least two replicate reactions must be positive. The predefined positive threshold was calculated as 10 SDs above the mean fluorescence of normal cattle brain homogenates. Previously described criteria were applied for classification of positive samples of RT-QuIC $(18,30,31)$.

\section{Mouse Bioassay}

To further characterize the potential infectivity of brain material from animal No. 1 and allow for comparison with RT-QuIC results, bioassays were conducted in TgBovXV (32) mice under isoflurane anesthesia as previously described (33). Inocula were derived from the brainstem of subclinical bovine No. 1 (10\% w/v in PBS) or the 
original classical BSE inoculum (1\% w/v in PBS) (34). Each mouse was inoculated with $20 \mu \mathrm{l}$ intracranially. Mice were monitored daily for the development of clinical signs by animal care staff. When signs suggestive of prion disease such as ataxia, listing or rolling gate, pelvic limb paresis, lethargy, or poor grooming with urine stained fur were recognizable by observation, animals were humanely euthanized and their brains prepared for analysis by EIA as described above. The attack rate was defined as the percentage of mice from an experimental group that had a positive EIA result. Incubation periods are expressed as the mean days postinoculation (days PI) for mice with positive EIA results. All animals that died due to intercurrent disease or without the development of clinical signs 2 SDs or less prior to the average incubation time were included in the calculation of attack rate. Using these criteria to calculate attack rate, 18 mice were used for the classical BSE group and 16 mice were included in the No. 1 inoculation group.

\section{RESULTS}

\section{Incubation Periods and Clinical Signs}

The incubation period for classical BSE in cattle is reported here as the time from inoculation to the time when unequivocal signs of clinical disease were present. Clinical signs of disease included abnormalities in gait or stance, moderate to severe ataxia, and hyperreaction to stimuli such as noise or movement. The average incubation period was approximately 22 months (22). One animal (No. 1) was euthanized at the termination of the study at 31 months without developing clinical signs (Table 1) despite receiving the same inoculum by intracranial inoculation without any noted deviations in procedure. Tissues from this animal were subjected to additional studies in an effort to explain the failure to develop clinical signs.

\section{Microscopic Examination and IHC}

In the cattle clinically affected with $\mathrm{BSE}, \mathrm{PrP}^{\mathrm{Sc}}$ immunoreactivity was detected in all brain regions examined and corresponded with spongiform change. Spongiform change was most severe in the brainstem (nucleus of the solitary tract and nucleus of the spinal tract of the trigeminal nerve (Figure 1A1), caudal midbrain, and hypothalamus. $\mathrm{PrP}^{\mathrm{Sc}}$ immunolabeling in the steer No. 2 (clinically affected with BSE) included granular, linear, aggregated, intraneuronal, and stellate labeling types. There was moderately strong immunolabeling of the cerebrum (neocortex) with prominent stellate and intraglial labeling and less granular and intraneuronal labeling compared to the thalamus, midbrain) and brainstem (Figure 1A2). There was no evidence of spongiform change or $\mathrm{PrP}^{\mathrm{Sc}}$ immunoreactivity in any of the other groups represented: inoculated with the BSE agent but prior to the onset of clinical signs (steer No. 1, Figures 1B1,B2); knockout cattle $\left(P R N P^{-/-}\right)$inoculated with the TME agent, or a sham-inoculated control animal (Figures 1C1,C2).

\section{Western Blotting in Brain Samples from Clinical, Subclinical, Knockout, and Sham- Inoculated Cattle}

Samples of brainstem at the level of the obex were collected from representative cattle. WBs were done using the monoclonal antibody $6 \mathrm{H} 4$ to demonstrate $\mathrm{PrP}^{\mathrm{Sc}}$. WB results are shown in Figure 2. WB confirmed evidence of $\mathrm{PrP}^{\mathrm{Sc}}$ in cattle with clinical signs (Nos. 2 and 3), but failed to demonstrate $\operatorname{PrP}^{\mathrm{Sc}}$ in cattle necropsied prior to the onset of clinical signs (No. 1), knockout cattle inoculated with ME (Nos. 4 and 5), or a sham-inoculated steer (No. 6).

\section{Quantitation of PrPsc by EIA in Brain Samples from Clinical, Subclinical, Knockout, and Sham-Inoculated Cattle}

Enzyme immunoassay was initially performed to on brainstem samples from all BSE-inoculated cattle to determine the relative amounts of misfolded prion protein present. Similar to IHC and $\mathrm{WB}$, no misfolded prion protein was detected in the brainstem of steer No. 1, whereas high levels were detected in the brainstem of cattle with clinical signs (steers No. 2 and No. 3; see Table 2).

Steer No. 1 was inoculated with brain homogenate of known infectivity, but brainstem samples tested negative by EIA. Therefore, EIA was performed on additional brain regions including cerebrum, cerebellum, midbrain, and thalamus. Samples from cerebrum and cerebellum also showed low OD similar to the negative control. Interestingly, relatively high levels of $\mathrm{PrP}^{\mathrm{sc}}$ were detected in the midbrain and thalamus regions (Table 2). When the same brain regions were tested from cattle with clinical signs, they were strongly positive by EIA. None of the brain regions sampled from the sham-inoculated control or knockout animals inoculated with TME were positive by EIA.

\section{RT-QuIC Seeding Activity in Brainstem Samples from Clinical, Subclinical, and Sham-Inoculated Control Cattle}

To investigate whether RT-QuIC can detect prion seeding activity from cattle prior to the onset of clinical signs of classical BSE, we compared brainstem homogenates from each of the experimental groups using the RT-QuIC assay with full length bovine $\operatorname{rPrP}$ [a.a. 25-241] substrate and the reaction conditions $(0.1 \mathrm{mg} / \mathrm{ml} \mathrm{rPrP}$ final concentration, $300 \mathrm{mM} \mathrm{NaCl}$, and $0.001 \%$ SDS) as previously used (29). An increase in ThT fluorescence was observed within $40 \mathrm{~h}$ incubation in each quadruplicate reaction seeded with $10^{-4}$ dilution of $10 \% \mathrm{w} / \mathrm{v}$ classical BSE brain homogenate including animal No. 2 and No. 3 (Figure 3). However, fluorescence was not produced in assays seeded with either brainstem of steer No. 1 or negative control animals indicating extremely low or absent prion levels in the brainstem of those animals.

\section{RT-QuIC Seeding Activity in Various Brain Regions of Cattle with Subclinical BSE}

In order to determine whether RT-QuIC can detect $\mathrm{PrP}^{\mathrm{Sc}}$ in other brain regions of subclinical cattle, samples from multiple brain regions of a steer No. 1 were assessed. We compared 5 brain regions including brainstem at the level of the obex, cerebrum, cerebellum, midbrain, and thalamus to a negative control sample. As can be seen in Figure 4A, all reactions seeded with the four brain regions (except brainstem) gave strong positive responses within $60 \mathrm{~h}$ for the $10^{-2}$ brain dilutions. The reaction seeded with thalamus had the shortest lag time about $25 \mathrm{~h}$. Reactions seeded 


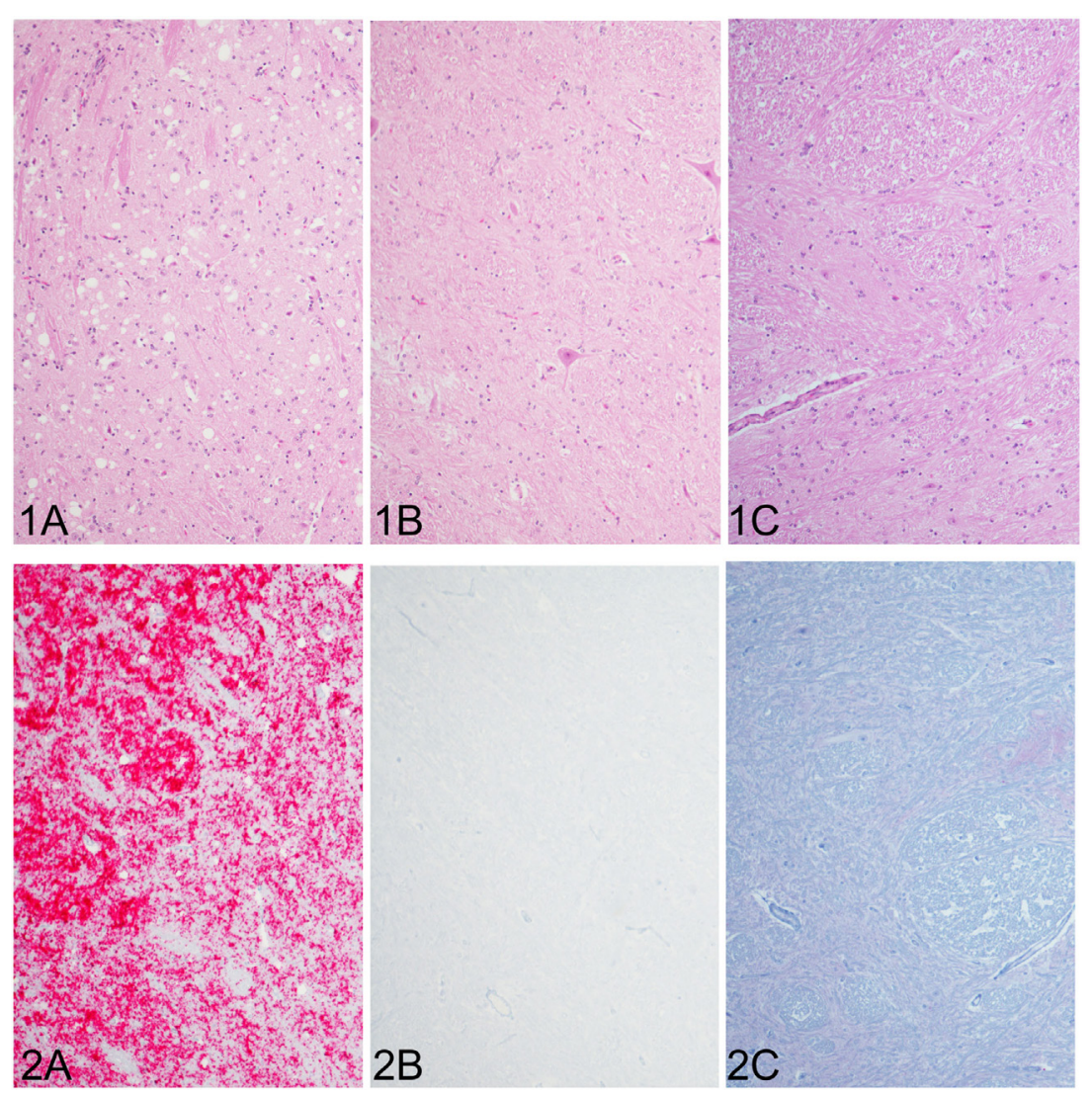

FIGURE 1 | Comparison of spongiform change and PrPsc accumulation in the brainstem at the level of the obex (all images of spinal tract and nucleus of the trigeminal nerve). Spongiform change is severe in the sections of brain steer No. 2 that had clinical signs of bovine spongiform encephalopathy (BSE) (1A) and corresponds with intense immunoreactivity for PrPsc [red chromogen; (2A)]. Neither spongiform change nor PrPsc immunostaining was observed in brain sections of a steer No. 1 with subclinical BSE (1B,2B), which appear similar to a sham-inoculated, negative control steer (1C,2C). Original magnification of all images is $10 \times$. Immunohistochemistry done with mAb F99/97.6.1.

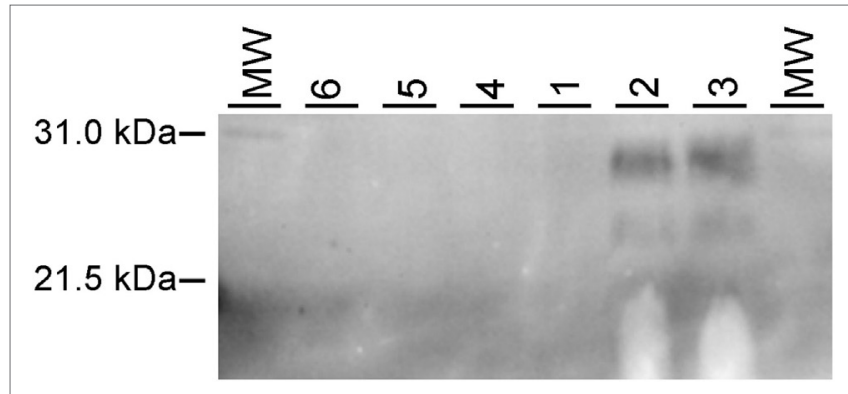

FIGURE 2 | Western blot analysis of relative amounts of $\mathrm{PrPsc}^{\mathrm{s}}$ in brain homogenates from bovine spongiform encephalopathy (BSE)-infected cattle. Each lane represents proteinase $\mathrm{K}(\mathrm{PK})$-digested brain homogenate probed with an anti-PrP monoclonal antibody 6H4. MW, molecular weight marker. Lanes are labeled with animal number: 6-sham-inoculated, 5 and 4-TMEinoculated knockout cattle, 1-steer with subclinical BSE, and 2 and 3cattle with clinical evidence of BSE.

with cerebrum and cerebellum also produced an increase in ThT fluorescence (Figure 4A) despite having OD values similar to the negative control of the EIA kit confirming that RT-QuIC is more sensitive than the EIA assay for prion detection. For the $10^{-4}$ brain dilution (see Figure 4B), a thalamus sample resulted in ThT fluorescence while assays with other brain regions showed weak or negative ThT responses.

\section{RT-QuIC Seeding Activity of Samples from Cattle with Clinical Signs of Classical BSE}

To further compare RT-QuIC reactions of steer No. 1 with those of cattle clinically ill with BSE, we tested brain samples from multiple regions. Table 1 summarizes characteristics of two animals including incubation period, inoculum source, and clinical signs. RT-QuIC detection of prion seeding activity from each brain region from cattle with clinical signs of BSE is demonstrated in Figure 5. RT-QuIC reaction mixtures were seeded with $10^{-2}$ dilutions of brain tissues including brainstem at the level of the obex, cerebrum, cerebellum, midbrain, and thalamus of either animal No. 2 (Figure 5A) or No. 3 (Figure 5B). As can be seen in Figure 5, reactions seeded with brainstem at the level of the obex from animals with clinical signs of BSE had a relatively shorter lag time than other reactions seeded with any other brain sections. Reactions seeded with samples from either the thalamus or the 
TABLE 2 | IDEXX HerdChek enzyme immunoassay results of various brain regions from experimental animals.

\begin{tabular}{|c|c|c|c|c|c|}
\hline Animal no. & OD for brainstem & OD for cerebrum & OD for cerebellum & OD for midbrain & OD for thalamus \\
\hline 1 (Subclinical) & 0.065 & 0.115 & 0.116 & 2.53 & 3.26 \\
\hline 2 (Clinical) & 3.3 & 1.6 & 1.3 & 4.0 & 0.28 \\
\hline 3 (Clinical) & 2.5 & 0.19 & 0.19 & 2.2 & 4.0 \\
\hline $4(\mathrm{KO})$ & 0.08 & 0.08 & 0.07 & 0.075 & 0.077 \\
\hline $5(\mathrm{KO})$ & 0.075 & 0.073 & 0.073 & 0.077 & 0.073 \\
\hline 6 (Sham-inoculated) & 0.08 & 0.068 & 0.062 & 0.073 & 0.069 \\
\hline
\end{tabular}

Positive cutoff value defined as 0.15. All positive samples are highlighted in blue.

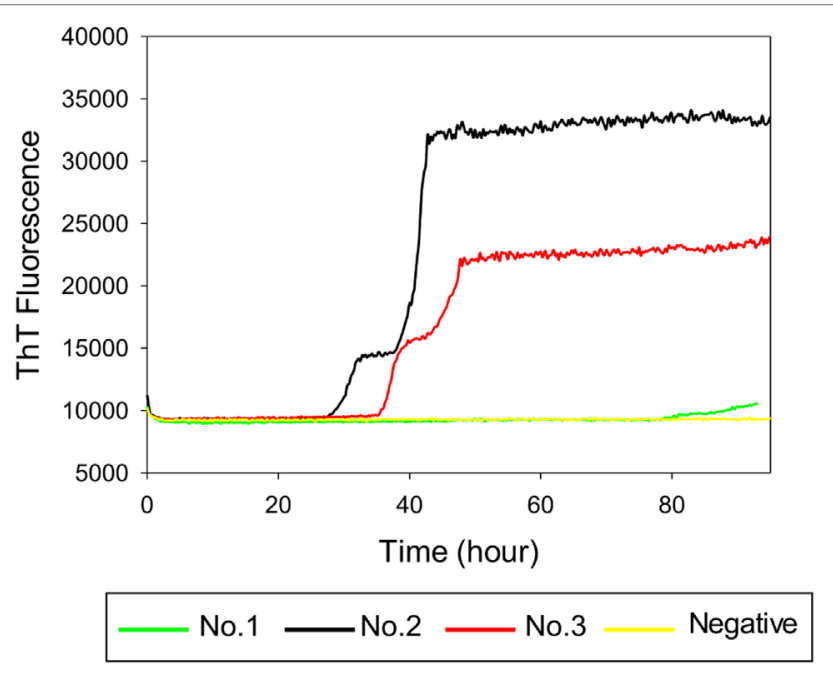

FIGURE 3 | Real-time quaking-induced conversion (RT-QulC) detection of classical bovine spongiform encephalopathy prion seeding activity using recombinant bovine prion protein. RT-QulC reaction mixtures were seeded with $10^{-4}$ dilutions of brain homogenate from classical BSE-affected cattle (animal No. 2 and No. 3), steer No. 1, or a negative control animal. A final SDS concentration of $0.001 \%$ in combination with $300 \mathrm{mM} \mathrm{NaCl}$ was used with the substrate. Data are presented as mean thioflavin T (ThT)

fluorescence of eight repeated reactions. The positive threshold was calculated as $\sim 10,000$ relative fluorescence units of brain homogenate from normal cattle.

midbrain had slightly longer lag times than reactions seeded with samples from the brainstem. Reactions seeded with samples from the cerebrum and the cerebellum also produced ThT fluorescence with longer lag time than midbrain or thalamus seeded reactions. All reactions seeded with negative brain failed to demonstrate increases in ThT fluorescence.

\section{PK Sensitivity PrPsc from the Brains of Cattle Inoculated with Classical BSE}

Samples from steer No. 1 were negative by IHC and WB, but positive in midbrain and thalamus using an EIA method that binds misfolded proteins rather than testing for protease resistance. Therefore, we wanted to test $\operatorname{PrP}^{\mathrm{Sc}}$ from the midbrain and thalamus of steer No. 1 for potential PK sensitivity. PK sensitivity testing was performed by measuring the absorbance of bound, misfolded protein before and after treatment with PK using EIA as described above. Brain homogenates of thalamus region from steers No. 1 and No. 2 were prepared in PBS and treated with various concentrations of PK $(0-0.5 \mathrm{mg} / \mathrm{ml})$. The results demonstrated that stable absorbance values after treatment with increasing amounts of PK in all samples (Figure 6).

\section{Comparison of RT-QuIC Seeding Activity of Samples from Cattle with Subclinical BSE to Samples from TME-Inoculated PRNP $^{-/-}$Knockout Cattle}

To test whether the ThT fluorescence response of samples from the midbrain and thalamus of steer No. 1 resulted from the residual inoculum, we measured RT-QuIC reactions seeded with brains from knockout $\left(P R N P^{-/-}\right)$cattle that were intracranially inoculated with TME. We evaluated whether different brain regions of intracranially inoculated knockout animals produced similar RT-QuIC responses to steer No. 1. Prion seeding activity was not detected in any RT-QuIC reactions seeded with brain samples from PRNP knockout cattle, whereas assays with brainstem samples from cattle with clinical signs of BSE or TME demonstrate strong ThT fluorescence (Figure 7).

\section{Mouse Bioassay}

To allow for comparison of RT-QuIC results with that of bioassay, we inoculated mice with the original classical BSE inoculum or brain homogenate from subclinical steer No. 1. Mice inoculated with $1 \%$ classical BSE inoculum $(18 / 18 ; 100 \%)$ developed clinical signs of difficulty moving and poor coordination and were euthanized with a mean incubation period of 299 days. Mice inoculated with $10 \%$ brainstem homogenate from steer \#1 developed clinical similar signs $(16 / 16 ; 100 \%)$, but with a longer mean incubation period of 363 days despite receiving a more concentrated brain homogenate (Figure 8).

\section{DISCUSSION AND CONCLUSION}

With this study, we provide proof of concept that RT-QuIC is a highly useful method for the detection of BSE prions in the brains of cattle with subclinical BSE. In the current study, an animal inoculated with classical BSE (No. 1) did not develop clinical illness and tested negative by standard diagnostics performed on brainstem despite a prolonged observation period of 31 months. When brainstem tested negative by EIA, we tested additional brain regions by EIA and RT-QuIC and compared the results to those of animals clinically ill with BSE in an effort to explain the failure of this animal to develop clinical signs. 


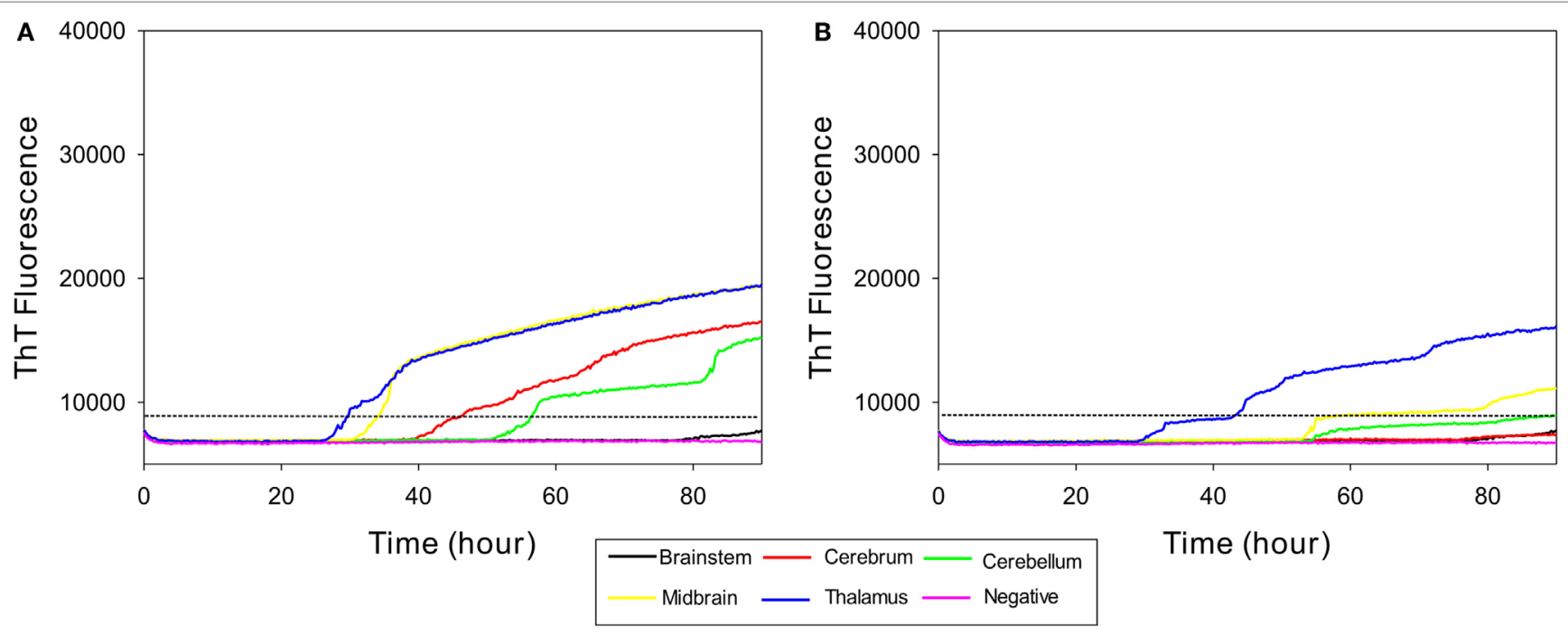

FIGURE 4 | Real-time quaking-induced conversion (RT-QulC) detection of prion seeding activity from each section of brain of a subclinical animal. Bovine recombinant prion protein was used as a substrate for RT-QulC reactions. RT-QulC reaction mixtures were seeded with $10^{-2}(\mathbf{A})$ and $10^{-4}$ (B) dilutions of brain tissues including brainstem, cerebrum, cerebellum, midbrain, and thalamus. A final SDS concentration of $0.001 \%$ in combination with 300 mM NaCl was used with the substrate. Data are presented as mean thioflavin T (ThT) fluorescence of quadruplicate reactions. The positive threshold was calculated as $\sim 10,000$ relative fluorescence units of brain homogenate from normal cattle.
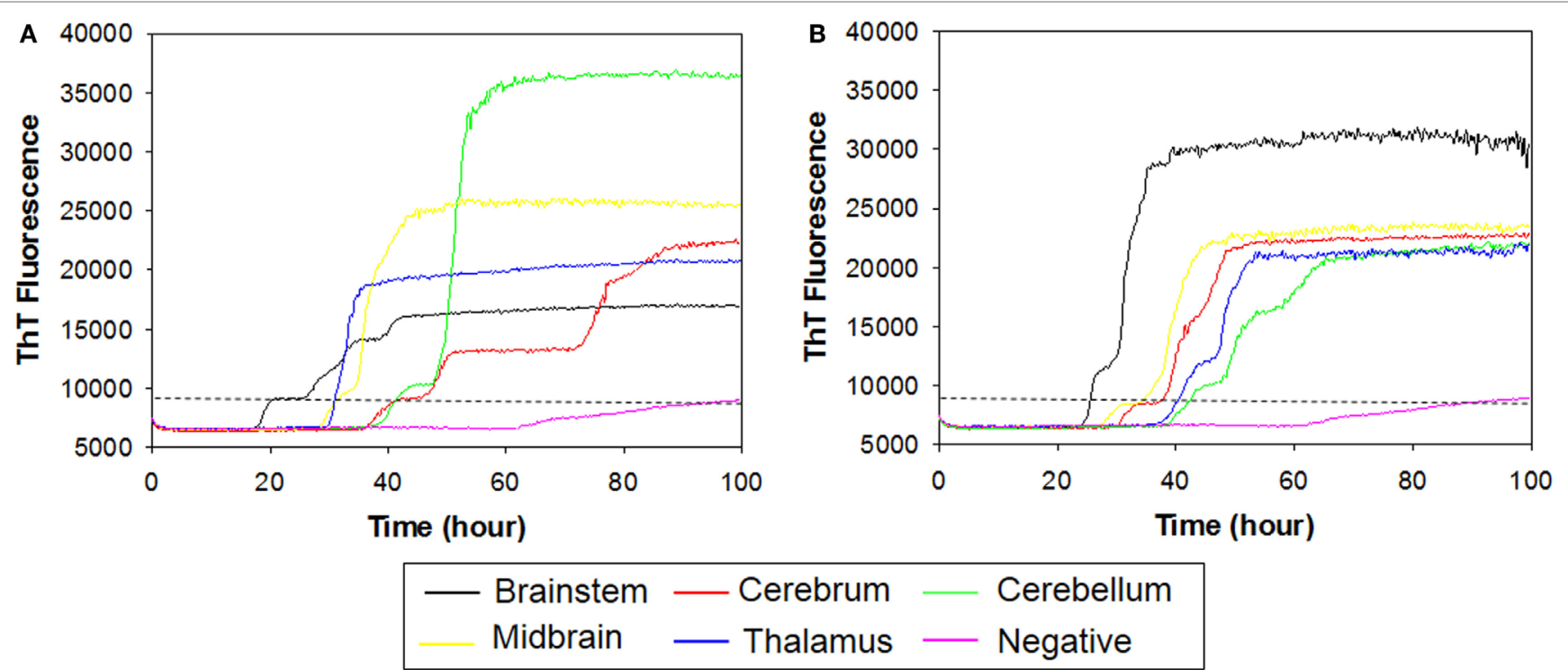

FIGURE 5 | Real-time quaking-induced conversion (RT-QulC) detection of prion seeding activity from each brain region of positive animals including animal No. 2 and No. 3. Recombinant bovine prion protein was used as a substrate for all RT-QulC reactions. RT-QulC reaction mixtures were seeded with $10^{-2}$ dilutions of brain tissues including brainstem, cerebrum, cerebellum, midbrain, and thalamus of either animal No. 2 (A) or No. 3 (B). A final SDS concentration of 0.001\% in combination with $300 \mathrm{mM} \mathrm{NaCl}$ was used with the substrate. Data are presented as mean thioflavin T (ThT) fluorescence of eight repetitions. The positive threshold was calculated as $\sim 10,000$ relative fluorescence units of brain homogenate from normal cattle.

Prion distribution differences between subclinical and clinical animals led us to perform additional RT-QuIC reactions in PRNP knockout animals to ensure our RT-QuIC results were not due to the presence of residual TSE inoculum. Prions were not detected in brain samples from $P R N P$ knockout animals by WB, EIA, IHC, or RT-QuIC.
In the current study, RT-QuIC detected lower levels of prions than traditional diagnostic tools. IHC and WB failed to identify any $\operatorname{PrP}^{S c}$ in the subclinical animal (No. 1), while testing multiple brain regions by EIA and RT-QuIC allowed us to detect misfolded protein in midbrain and thalamus. Further, we were able to detect seeding activity in brain regions including cerebrum and 


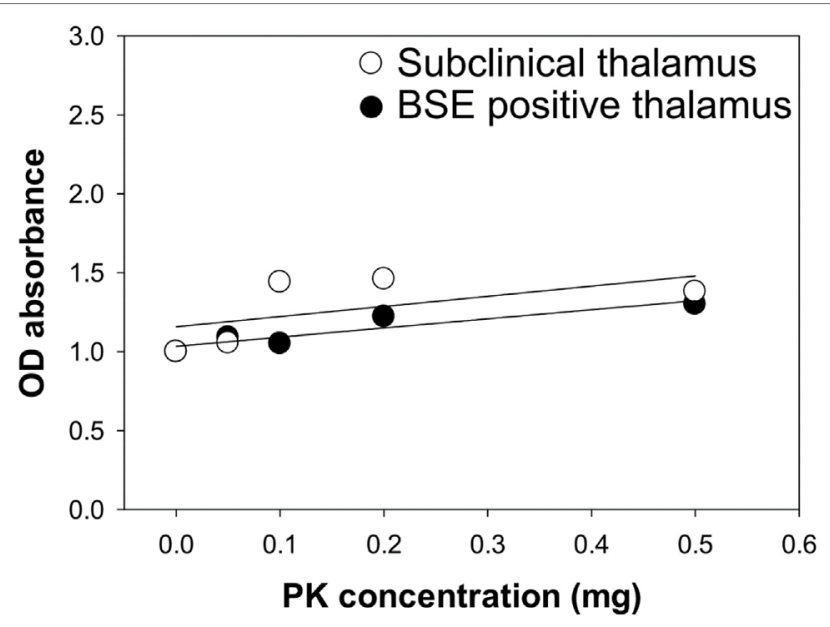

FIGURE 6 | Assessment of proteinase $\mathrm{K}$ (PK) sensitivity of PrPsc from the brains of bovine spongiform encephalopathy (BSE)-inoculated cattle. Brain samples from a subclinical animal (open circle) and animal with clinical signs of BSE (closed circle) were treated with increasing concentrations of PK.

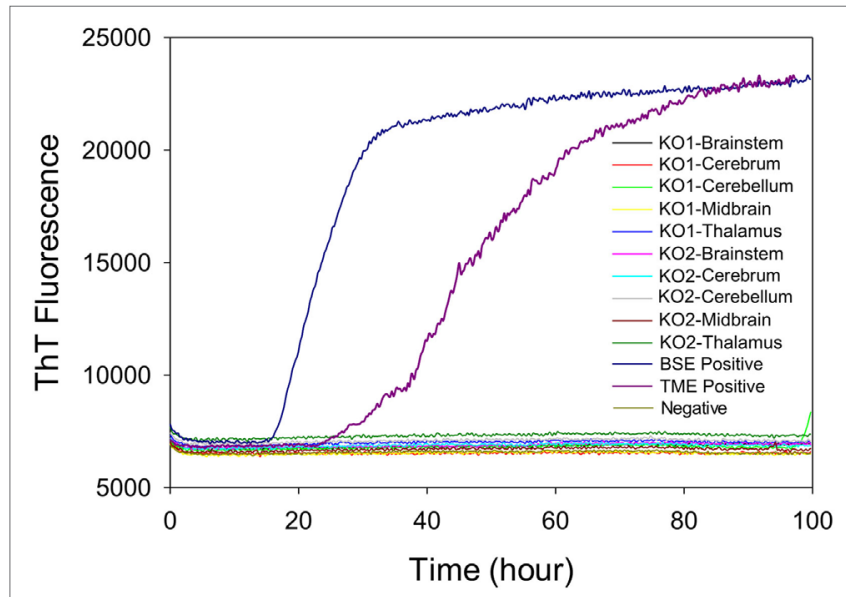

FIGURE 7 | Comparison of real-time quaking-induced conversion reactions using different brain regions of samples from prion knockout $\left(P R N P^{-/-}\right)$ animals or negative or positive controls. A final SDS concentration of $0.001 \%$ in combination with $300 \mathrm{mM} \mathrm{NaCl}$ was used with the substrate. Data are presented as mean thioflavin T (ThT) fluorescence of quadruplicate reactions. The positive threshold was calculated as $\sim 10,000$ relative fluorescence units of brain homogenate from normal cattle.

cerebellum that were negative by EIA. This result is consistent with a recent study that demonstrated that RT-QuIC is more sensitive for the detection of $\mathrm{PrP}^{\mathrm{CWD}}$ during early infection than tyramide signal amplification-IHC (35).

Real-time quaking-induced conversion has been successfully used for sensitive and specific detection of prion diseases for humans and animals $(11-13,15-17,36)$. We previously reported different types of BSE prions can be detected and differentiated using RT-QuIC with recombinant bovine prion protein (29), and the previous work of others evaluated detection and discrimination of classical, H-, and L-BSE prions by full-length

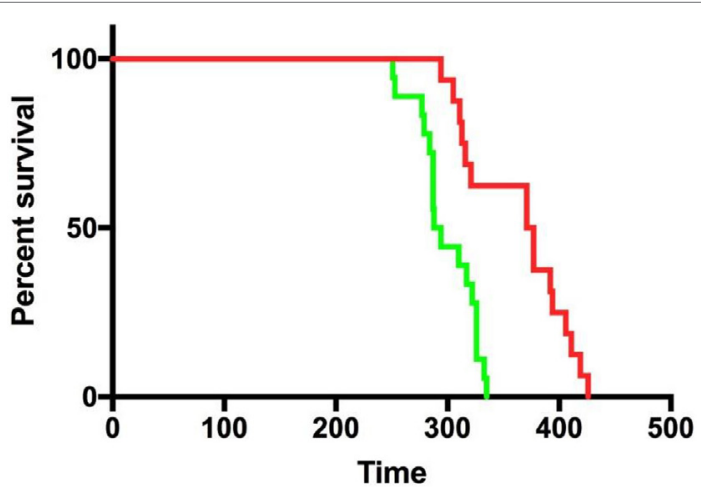

Animal No. $1 \rightarrow$ BSE-C

FIGURE 8 | Infectivity was present in the brainstem of steer No. 1 despite testing negative by immunohistochemistry, Western blot, and real-time quaking-induced conversion. TgBov XV mice inoculated with a 1\% homogenate of the original classical bovine spongiform encephalopathy (BSE) inoculum had $100 \%$ attack rate and a mean incubation period of 299 days (green line). Mice inoculated with a 10\% homogenate from the brainstem of the subclinical steer No. 1 also had a 100\% attack rate but with a prolonged mean incubation period of 363 days (red line). The attack rate was defined as the percentage of mice that had a positive enzyme immunoassay (EIA) result from an experimental group that includes all mice that died within 2 SDs of the mean incubation period. Incubation periods are expressed as the mean days postinoculation (days PI) for mice with positive EIA results.

chimeric hamster-sheep prion proteins, $\mathrm{N}$-terminally truncated hamster prion protein [90-231] (37), full-length sheep protein, and full-length bank vole protein (a.a. 23-230). There has been significant effort to detect prions in presymptomatic diagnostic samples including CSF, blood, urine, saliva, and nasal brushings by RT-QuIC [7-16]. CWD prions were detected in urine collected from presymptomatic deer and in fecal extracts by using RT-QuIC (38) suggesting potential applications in CWD surveillance and control.

In the present study, there were differences in prion distribution between the brains of subclinical and clinically affected cattle after intracranial BSE inoculation. In the subclinically affected steer (No. 1), the highest levels of prions accumulated in the thalamus and midbrain, while no prions were detected in the brainstem. Most likely this reflects prion accumulation in the location that the inoculum was deposited without further spread into other brain regions. A study comparing preclinical cattle infected naturally with BSE to clinically affected cattle either naturally or experimentally infected with BSE by the oral route found the most abundant $\mathrm{PrP}^{\mathrm{Sc}}$ in the brainstem area (39), which is consistent with ascension to the brain from the gut by sympathetic and parasympathetic projections (40). In our experiment, abundant prions were observed in the brainstem of cattle with clinical signs of BSE, which is similar to the amount in their thalamus or midbrain regions. Interestingly, prions in the brainstem of cattle with clinical evidence of BSE seeded the RT-QuIC reactions faster than any other brain region despite the brainstem area having lower EIA OD values (Table 2) in comparison to other brain regions. This suggests that higher concentrations of prions 
do not necessarily seed the reaction faster. Perhaps prions of the brainstem exist in a preferred conformation for better conversion despite being present in lower concentrations.

Bioassay of brainstem from steer No. 1 confirmed infectivity and RT-QuIC results despite being negative by IHC, WB, and EIA. Bioassay was performed with a $10 \%$ homogenate as compared to $1 \%$ for the original classical BSE inoculum, yet the average incubation period longer confirming lower infectivity in the brainstem of the subclinical steer. We previously reported that the retinas of animals inoculated with the agent of classical BSE become substantially thinner than both their baseline (preinoculation) thickness and the thickness of sham-inoculated control animals (22). This decrease in retinal thickness preceded the onset of clinical signs by an average of 10 months. It is interesting to note that in the previous study steer No. 1 had a substantially decreased retinal thickness value at 12 months postinoculation and would have been classified as "positive" by these previously reported criteria (22).

After being observed for nearly 9 months longer than other cattle in the same experimental group, a steer intracranially inoculated with classical BSE was euthanized without demonstrating clinical signs suggestive of prion disease. WB and IHC failed to demonstrate $\mathrm{PrP}^{\mathrm{Sc}}$ in any region of the brain. In an effort to investigate the reason that this animal failed to develop clinical signs, further studies were conducted using RT-QuIC, which demonstrated high levels of prions in the thalamus and midbrain. In summary, we demonstrate that RT-QuIC can be useful to detect prions in cattle with subclinical BSE and that BSE prions can be differently distributed in the brain regions as incubation progresses.

\section{ETHICS STATEMENT}

The animal experiments were carried out in accordance with the Guide for the Care and Use of Laboratory Animals (Institute of

\section{REFERENCES}

1. Prusiner SB. Prions. Proc Natl Acad Sci U S A (1998) 95(23):13363-83. doi:10.1073/pnas.95.23.13363

2. Collinge J. Prion diseases of humans and animals: their causes and molecular basis. Annu Rev Neurosci (2001) 24:519-50. doi:10.1146/annurev. neuro.24.1.519

3. Weissmann C. The ninth Datta lecture. Molecular biology of transmissible spongiform encephalopathies. FEBS Lett (1996) 389(1):3-11. doi:10.1016/0014-5793(96)00610-2

4. Bradley R, Wilesmith JW. Epidemiology and control of bovine spongiform encephalopathy (BSE). Br Med Bull (1993) 49(4):932-59. doi:10.1093/oxfordjournals.bmb.a072654

5. Smith PG, Bradley R. Bovine spongiform encephalopathy (BSE) and its epidemiology. Br Med Bull (2003) 66:185-98. doi:10.1093/bmb/66.1.185

6. Van Everbroeck B, Pals P, Martin JJ, Cras P. Antigen retrieval in prion protein immunohistochemistry. J Histochem Cytochem (1999) 47(11):1465-70. doi:10.1177/002215549904701112

7. Peretz D, Williamson RA, Matsunaga Y, Serban H, Pinilla C, Bastidas RB, et al. A conformational transition at the $\mathrm{N}$ terminus of the prion protein features in formation of the scrapie isoform. J Mol Biol (1997) 273(3):614-22. doi:10.1006/jmbi.1997.1328

8. Soto C, Saborio GP, Anderes L. Cyclic amplification of protein misfolding: application to prion-related disorders and beyond. Trends Neurosci (2002) 25(8):390-4. doi:10.1016/S0166-2236(02)02195-1
Laboratory Animal Resources, National Academy of Sciences, Washington, DC, USA) and the Guide for the Care and Use of Agricultural Animals in Research and Teaching (Federation of Animal Science Societies, Champaign, IL, USA). The protocols were approved by the Institutional Animal Care and Use Committee at the National Animal Disease Center (protocol numbers: 3636 and 3985).

\section{AUTHOR CONTRIBUTIONS}

Conceived and designed the experiments: SH, MG, and JG. Performed the experiments: SH and JG. Analyzed the data: SH, MG, EN, and JG. Contributed reagents/materials/analysis tools: AB-B, MG, and JG. Wrote the article: SH, MG, and JG. All authors read and approved the final manuscript.

\section{ACKNOWLEDGMENTS}

The authors thank Semakaleng Lebepe-Mazur, Trudy Tatum, Kevin Hassall, Joe Lesan, and Leisa Mandell for providing technical support to this project. Mention of trade names or commercial products in this article is solely for the purpose of providing specific information and does not imply recommendation or endorsement by the US Department of Agriculture. USDA is an equal opportunity provider and employer.

\section{FUNDING}

This research was funded in its entirety by congressionally appropriated funds to the United States Department of Agriculture, Agricultural Research Service. The funders of the work did not influence study design, data collection and analysis, decision to publish, or the preparation of the manuscript.

9. Soto C, Anderes L, Suardi S, Cardone F, Castilla J, Frossard MJ, et al. Presymptomatic detection of prions by cyclic amplification of protein misfolding. FEBS Lett (2005) 579(3):638-42. doi:10.1016/j.febslet.2004.12.035

10. Saa P, Castilla J, Soto C. Cyclic amplification of protein misfolding and aggregation. Methods Mol Biol (2005) 299:53-65. doi:10.1074/jbc.M603964200

11. Wilham JM, Orru CD, Bessen RA, Atarashi R, Sano K, Race B, et al. Rapid end-point quantitation of prion seeding activity with sensitivity comparable to bioassays. PLoS Pathog (2010) 6(12):e1001217. doi:10.1371/journal. ppat.1001217

12. Atarashi R, Satoh K, Sano K, Fuse T, Yamaguchi N, Ishibashi D, et al. Ultrasensitive human prion detection in cerebrospinal fluid by real-time quaking-induced conversion. Nat Med (2011) 17(2):175-8. doi:10.1038/ nm. 2294

13. Atarashi R, Sano K, Satoh K, Nishida N. Real-time quaking-induced conversion: a highly sensitive assay for prion detection. Prion (2011) 5(3):150-3. doi:10.4161/pri.5.3.16893

14. Corsaro A, Thellung S, Bucciarelli T, Scotti L, Chiovitti K, Villa V, et al. High hydrophobic amino acid exposure is responsible of the neurotoxic effects induced by E200K or D202N disease-related mutations of the human prion protein. Int J Biochem Cell Biol (2011) 43(3):372-82. doi:10.1016/j. biocel.2010.11.007

15. McGuire LI, Peden AH, Orru CD, Wilham JM, Appleford NE, Mallinson G, et al. Real time quaking-induced conversion analysis of cerebrospinal fluid in sporadic Creutzfeldt-Jakob disease. Ann Neurol (2012) 72(2):278-85. doi:10.1002/ana.23589 
16. Orru CD, Wilham JM, Vascellari S, Hughson AG, Caughey B. New generation QuIC assays for prion seeding activity. Prion (2012) 6(2):147-52. doi:10.4161/ pri. 19430

17. Peden AH, McGuire LI, Appleford NE, Mallinson G, Wilham JM, Orru CD, et al. Sensitive and specific detection of sporadic Creutzfeldt-Jakob disease brain prion protein using real-time quaking-induced conversion. J Gen Virol (2012) 93(Pt 2):438-49. doi:10.1099/vir.0.033365-0

18. Dassanayake RP, Orru CD, Hughson AG, Caughey B, Graca T, Zhuang D, et al. Sensitive and specific detection of classical scrapie prions in the brains of goats by real-time quaking-induced conversion. J Gen Virol (2016) 97(3):803-12. doi:10.1099/jgv.0.000367

19. Hamir AN, Miller JM, Stack MJ, Chaplin MJ. Failure to detect abnormal prion protein and scrapie-associated fibrils $6 \mathrm{wk}$ after intracerebral inoculation of genetically susceptible sheep with scrapie agent. Can J Vet Res (2002) 66(4):289-94.

20. Greenlee JJ, Nicholson EM, Smith JD, Kunkle RA, Hamir AN. Susceptibility of cattle to the agent of chronic wasting disease from elk after intracranial inoculation. J Vet Diagn Invest (2012) 24(6):1087-93. doi:10.1177/1040638712461249

21. Richt JA, Kunkle RA, Alt D, Nicholson EM, Hamir AN, Czub S, et al. Identification and characterization of two bovine spongiform encephalopathy cases diagnosed in the United States. J Vet Diagn Invest (2007) 19(2):142-54. doi: $10.1177 / 104063870701900202$

22. West Greenlee MH, Smith JD, Platt EM, Juarez JR, Timms LL, Greenlee JJ. Changes in retinal function and morphology are early clinical signs of disease in cattle with bovine spongiform encephalopathy. PLoS One (2015) 10(3):e0119431. doi:10.1371/journal.pone.0119431

23. Cutlip RC, Miller JM, Race RE, Jenny AL, Katz JB, Lehmkuhl HD, et al. Intracerebral transmission of scrapie to cattle. J Infect Dis (1994) 169(4): 814-20. doi:10.1093/infdis/169.4.814

24. Hamir AN, Miller JM, Kunkle RA, Hall SM, Richt JA. Susceptibility of cattle to first-passage intracerebral inoculation with chronic wasting disease agent from white-tailed deer. Vet Pathol (2007) 44(4):487-93. doi:10.1354/vp.44-4-487

25. Hamir AN, Kunkle RA, Miller JM, Bartz JC, Richt JA. First and second cattle passage of transmissible mink encephalopathy by intracerebral inoculation. Vet Pathol (2006) 43(2):118-26. doi:10.1354/vp.43-2-118

26. Greenlee JJ, Smith JD, West Greenlee MH, Nicholson EM. Clinical and pathologic features of H-type bovine spongiform encephalopathy associated with E211K prion protein polymorphism. PLoS One (2012) 7(6):e38678. doi:10.1371/journal.pone.0038678

27. O’Rourke, Baszler et al. Preclinical diagnosis of scrapie by immunohistochemistry of third eyelid lymphoid tissue. J Clin Microbiol. (2000) 38(9):3254-9.

28. Vrentas CE, Onstot S, Nicholson EM. A comparative analysis of rapid methods for purification and refolding of recombinant bovine prion protein. Protein Expr Purif (2012) 82(2):380-8. doi:10.1016/j.pep.2012.02.008

29. Hwang S, Greenlee JJ, Nicholson EM. Use of bovine recombinant prion protein and real-time quaking-induced conversion to detect cattle transmissible mink encephalopathy prions and discriminate classical and atypical L- and H-type bovine spongiform encephalopathy. PLoS One (2017) 12(2):e0172391. doi:10.1371/journal.pone.0172391

30. Orru CD, Groveman BR, Hughson AG, Zanusso G, Coulthart MB, Caughey B. Rapid and sensitive RT-QuIC detection of human Creutzfeldt-Jakob disease using cerebrospinal fluid. MBio (2015) 6(1):e2451-2414. doi:10.1128/ mBio.02451-14
31. Orru CD, Bongianni M, Tonoli G, Ferrari S, Hughson AG, Groveman BR, et al. A test for Creutzfeldt-Jakob disease using nasal brushings. $N$ Engl J Med (2014) 371(6):519-29. doi:10.1056/NEJMoa1315200

32. Buschmann A, Pfaff E, Reifenberg K, Muller HM, Groschup MH. Detection of cattle-derived BSE prions using transgenic mice overexpressing bovine PrP(C). Arch Virol Suppl (2000) 16:75-86.

33. Smith JD, Nicholson EM, Greenlee JJ. Evaluation of a combinatorial approach to prion inactivation using an oxidizing agent, SDS, and proteinase K. BMC Vet Res (2013) 9:151. doi:10.1186/1746-6148-9-151

34. West Greenlee MH, Lind M, Kokemuller R, Mammadova N, Kondru N, Manne S, et al. Temporal resolution of misfolded prion protein transport, accumulation, glial activation, and neuronal death in the retinas of mice inoculated with scrapie. Am J Pathol (2016) 186(9):2302-9. doi:10.1016/j. ajpath.2016.05.018

35. Hoover CE, Davenport KA, Henderson DM, Denkers ND, Mathiason CK, Soto C, et al. Pathways of prion spread during early chronic wasting disease in deer. J Virol (2017) 91(10):e00077-17. doi:10.1128/JVI.00077-17

36. Elder AM, Henderson DM, Nalls AV, Wilham JM, Caughey BW, Hoover EA, et al. In vitro detection of prionemia in TSE-infected cervids and hamsters. PLoS One (2013) 8(11):e80203. doi:10.1371/journal.pone.0080203

37. Masujin K, Orru CD, Miyazawa K, Groveman BR, Raymond LD, Hughson AG, et al. Detection of atypical h-type bovine spongiform encephalopathy and discrimination of bovine prion strains by real-time quaking-induced conversion. JClin Microbiol (2016) 54(3):676-86. doi:10.1128/JCM. 02731-15

38. Cheng YC, Hannaoui S, John TR, Dudas S, Czub S, Gilch S. Early and noninvasive detection of chronic wasting disease prions in elk feces by real-time quaking induced conversion. PLoS One (2016) 11(11):e0166187. doi:10.1371/ journal.pone.0166187

39. Iwata N, Sato Y, Higuchi $\mathrm{Y}$, Nohtomi K, Nagata N, Hasegawa H, et al. Distribution of $\operatorname{PrP}(\mathrm{Sc})$ in cattle with bovine spongiform encephalopathy slaughtered at abattoirs in Japan. Jpn J Infect Dis (2006) 59(2):100-7.

40. Kaatz M, Fast C, Ziegler U, Balkema-Buschmann A, Hammerschmidt B, Keller M, et al. Spread of classic BSE prions from the gut via the peripheral nervous system to the brain. Am J Pathol (2012) 181(2):515-24. doi:10.1016/j. ajpath.2012.05.001

Disclaimer: Mention of trade names or commercial products in this publication is solely for the purpose of providing specific information and does not imply recommendation or endorsement by the US Department of Agriculture. USDA is an equal opportunity provider and employer.

Conflict of Interest Statement: The authors declare that the research was conducted in the absence of any commercial or financial relationships that could be construed as a potential conflict of interest.

Copyright (c) 2018 Hwang, West Greenlee, Balkema-Buschmann, Groschup, Nicholson and Greenlee. This is an open-access article distributed under the terms of the Creative Commons Attribution License (CC BY). The use, distribution or reproduction in other forums is permitted, provided the original author(s) or licensor are credited and that the original publication in this journal is cited, in accordance with accepted academic practice. No use, distribution or reproduction is permitted which does not comply with these terms. 